\title{
Component Separation with or without Mesh Application for the Repair of Large Ventral Abdominal Wall Incisional Hernia
}

\author{
Mohamed A. Aamer, MD. MRCS; ${ }^{1}$ Sherine Metwally, MD. ${ }^{2}$
}

\section{1) Department of General Surgery, Faculty of Medicine Ain Shams University.}

2) Department of Plastic Surgery, Faculty of Medicine Ain Shams University.

Background: Incisional hernia is a common long-term complication after abdominal surgery. Sound repair of large abdominal wall incisional hernia is required to be a tension free repair with low recurrence.

Methods: The current study comprised 82 consecutive patients with large ventral abdominal wall incisional hernia. The study was conducted in Ain Shams University Hospitals during the period from May 2011 till August 2014. The patients were randomly classified into 2 groups: Group I (42 patients) who underwent repair of their hernias by component separation technique without application of a mesh; group II (42 patients) who underwent repair of their hernias by the component separation technique with application of polypropylene mesh.

Results: Group I had the advantages of statistically significant less mean operative time (165 \pm 24.09 min. Compared to $195.60 \pm 19.42$ in group II) $(P<0.0001)$. There is less wound infection in group I (2 patients: $4.76 \%$ vs 5 patients in group II: 11.90\%) $(P=0.4326)$. There is less seroma without significant difference $(P=0.5700)$. Group II had the advantages of a statistically significant lower recurrence rate (1 recurrence in group $I \simeq 2.38 \%$, compared to 8 recurrences in group $I I \simeq 19.05 \%)(P=0.02294)$. There is less post-operative anemia in group II (2 patients $\simeq 4.76 \%$, compared to 4 patients in group $I \simeq 9.52 \%$ ) and less skin necrosis (2 patients in group $I I \simeq 4.76 \%$ vs 3 patients in group $I \simeq 7.14 \%$ ) but the difference is insignificant $(P=1)$. There was insignificant difference in the amount of intra-operative blood less $(P=0.1255)$, post-operative intra-abdominal pressure $(P=0.4733)$ and post-operative pain in 1 st 48 hours $(P=0.6675)$ between both groups. Group II had less pain in the $3 r d$ to 7 th post-operative day without statistical significance $(P=0.0758)$. There was no significant difference between both groups in the mean hospital stay and the mean time to return to work ( $P=1.000$ and 0.9596 respectively).

Conclusion: Mesh application after component separation is ideal for the repair of large ventral abdominal wall incisional hernias offering a tension free repair with lower recurrence rate.

\section{Introduction:}

Incisional hernia is a common long-term copmplication following abdominal surgery as it is estimated to occur in approximately $10 \%$ of cases. However the true incidence is probably higher since the majority are asymptomatic. ${ }^{1}$

The risk factors for the development of incisional hernia include obesity, diabetes, emergency surgery, post-operative wound dehiscence, smoking and post-operative wound infection..$^{2,3}$

After repair, these hernias have a recurrence rate that approaches 30 to $50 \%$ in some series. ${ }^{4}$ However, studies describing a tension-free closure using a prosthetic mesh patch report recurrence rates in the $10 \%$ range. ${ }^{5}$

Incisional hernias are classified according to the size (real fascial jap) into: Small $(<5$ $\mathrm{cm}$ in width or length), medium $(5-10 \mathrm{~cm}$ in width or length), and large $(>10 \mathrm{~cm}$ in width 
or length). 6

The component separation technique which was described by Ramirez and Colleagues, ${ }^{7}$ allows a flap of the rectus muscle, anterior rectus sheath and internal oblique transversus to be advanced in the mid line a maximum of $10 \mathrm{~cm}$ i.e. Incisional hernia gaps of $20 \mathrm{~cm}$ can be closed.

The external oblique is released from its attachment to the rectus muscle and a plane dissected between the external and internal oblique apponeuroses. An additional step is the complete release of the rectus abdomines muscle from its anterior and posterior sheaths by incising the posterior rectus sheath at its medial border. ${ }^{8}$ Figure (1).

This technique allows the tension less approximation of the rectus muscles in large and recurrent hernias precluding the problem of abdominal compartment syndrome. ${ }^{9}$

Many surgeons recommend the additional application of synthetic mesh in an on lay position to supplement the attenuated layers of the anterior abdominal wall. ${ }^{10}$

Major complications which can occur in repair of large incisional hernias include mesh infection and enterocutaneous fistula which may result in prolonged morbidity and require re-operation. ${ }^{8}$

Components separation represents a simple, one-stage technique for abdominal wall reconstruction that is associated with minimal donor site morbidity. This technique is especially recommended when the defects are too large or located too close to the xiploid process. ${ }^{11}$

The current study is a prospective randomized controlled clinical trial comparing between the technique of component separation with application of mesh and without application of a mesh for the repair of large ventral abdominal wall incisional hernia.

\section{Patients and methods:}

The current study comprised 84 consecutive patients with large ventral abdominal wall incisional hernias, chosen from those patients attending the outpatient clinics of Ain Shams University Hospitals during the period from
May 2011 till August 2014.

The patients were randomly classified into 2 groups by simple randomization: Group I: Included 42 patients who underwent repair of their incisional hernias by the component separation technique without application of a mesh.

Group II: Included 42 patients who underwent repair of their incisional hernia by the component separation technique followed by application of polypropylene mesh to supplement the repair.

Patients with obstructed or strangulated hernias were excluded from the study. Also those with Child C liver disease, cardiomyopathy and pregnant female patients were excluded from the study. Also patients with severe restrictive or obstructive air way disease were excluded.

On their enrollment into the study, all participating patients signed consents indicating their agreement to participate in the study and the follow-up visits.

Full history is taken from every patient including medical comorbidities, smoking and previous surgeries.

Full clinical examination including general and local abdominal examination were done.

Pre-operative investigations included full laboratory investigations including $(\mathrm{CBC}$, PT and PTT, liver function tests, kidney function tests, and fasting blood glucose), pelviabdominal U/S, ECG, echocardiograph, chest X-ray and pulmonary function tests.

The operation was performed under general or epidural/spinal anaesthesia according to the anaesthesia staff assessment.

The operation was performed by general and plastic surgeons to give the best chance for patients with large abdominal wall hernias.

Operative technique is as follows: A transverse suprapubic incision was used. Flaps were elevated with limiting the undermining under the costal margins. The hernial sac was dissected from the myoaponeurotic layers till the defect in the peritoneum was opened and adhesolysis between the intestinal contents and between them and the sac was done.

Then components separation was performed by incising the external oblique 
fascia and dissecting through the external oblique muscle until the internal oblique fascia was encountered. The external oblique muscle was then elevated to the level of mid axillary line on both sides. The rectus abdominis muscle was completely released from anterior and posterior sheaths by incising the posterior rectus sheath at its medial border. This allowed a flap of the rectus muscle, anterior rectus sheath and internal oblique and transversus abdominis muscles to be advanced in the midline for a maximum of $10 \mathrm{~cm}$ to cover the large hernial defect, then plication of the midline was done from the xiphoid process till the pubis. Figure (2).

In group II a polypropylene mesh of $30 \times 30$ size was used in an onlay fashion to reinforce the repair. It was fixed by nonabsorbable 2-0 polypropylene sutures.

In both groups, the excess skin and subcutaneous tissue was excised, the umbilicus was transposed to upper position as planed pre-operatively, 2 redivacs, of size 18 were left, and the fascia was closed in 2 layers, the skin was closed by subcuticular absorbable monofilament suture (Monocryl, 3-0).

The operative time, intra-operative blood loss and any intra-operative complications were recorded and compared between both groups of patients.

Post-operative intra-abdominal pressure was measured by manometers attached to urinary catheters and compared between both groups of patients post-operative pain was assessed by visual analogue scale and compared between both groups of patients. Any incidence of post-operative complications was recorded and compared between both groups of patients.

The mean hospital stay and mean time to return to work was assessed and compared between both groups of patients.

The patients were followed up in the outpatient clinic twice weekly in the first 2 weeks after discharge, then weekly for 1 month, then monthly for 6 months, and then every 3 months for 1 year.

During the follow-up visits any occurrence of complications or hernial recurrence was recorded and compared between both groups of patients.

Statistical Analysis:

The collected data was revised, coded, tabulated and introduced to a PC using statistical package for social science (SPSS 15.01 for Windows). The statistical significance was assessed using the Fisher's exact test for categorical data and the unpaired t-test for numerical data. P-value $<0.05$ was considered significant.

\section{Results:}

Group I comprised 25 females (59.52\%) and 18 males (40.48\%), while group II comprised 27 females (64.29\%) and 15 males (35.71\%) $(\mathrm{P}=0.6615337)$.

There was no difference in the mean age between both groups $(\mathrm{P}=0.05544)$, as the mean age for group I was $45.45 \pm 8.39$ years, and the mean age for group II was 48.97 \pm 8.23 years (Table 1 ).

The operation was performed under general anaesthesia in 32 patients of group I $(76.19 \%)$, and 34 patients in groups II $(80.95 \%)$, and under regional (spinal/ epidural) anaesthesia in 10 patients of group I (23.81\%) and in 8 patients of group II (19.05\%) $(\mathrm{P}=0.79093072)$.

The mean operative time was significantly shorter in group I (mean operative time 165 $\pm 24.09 \mathrm{~min}$.) than group II (mean operative time $195.60 \pm 19.42 \mathrm{~min}$. $)(\mathrm{P}<0.0001)$.

There was no significant difference in the mean amount of intra-operative blood loss between both group ( $\mathrm{P}=0.1255)$ Table (2).

There was no intra-operative complications in both groups of patients, except small intestinal injury in 1 patient of group I ( $2.38 \%)$ which was repaired in 2 layers without any subsequent leakage $(\mathrm{P}=1)$.

There was no statistically significant difference in the mean of the maximum recorded intra-abdominal pressure between both groups of patients. The mean maximum intra-abdominal pressure for group I was $(7.2 \pm 1.72 \mathrm{mmHg}$ ) (range $5-12 \mathrm{mmHg}$ ) and for group II it was (7.29 \pm 1.61$)$ (range 4-11 
$\mathrm{mmHg})(\mathrm{P}=0.4733)$ Table (3).

There was no manifestations of abdominal compartmental syndrome in any patients of both groups

There was a statistically insignificant difference in the post-operative pain severity between both groups during the $1^{\text {st }} 48$ hours $(\mathrm{P}=0.6675)$. From the 3 rd to the $7^{\text {th }}$ postoperative day the pain severity was less in group II than group I as the mean VAS for group II was $3.24 \pm 0.93$, while that for group I was $3.64 \pm 1.12$. However the difference did not reach statistical significance $(\mathrm{P}=0.0758)$ Table (3).

There was no statistically significant difference between both groups regarding the mean hospital stay $(6.50 \pm 1.33$ days in group I, $6.50 \pm 1.84$ days in group II) $(\mathrm{P}=1)$, and the mean time to return to work $(23.17 \pm 4.46$ days in group I, 23.12 \pm 4.17 days in group II) $(\mathrm{P}=0.9596)$ Table (4).

Comparing the post-operative complications between both groups of patients revealed the following results:

1. Anaemia corrected by blood transfusion occurred in 4 patients of group I $(9.52 \%)$ and 2 patients of group II $(4.76 \%)$. Neither of these patients required re-operation $(\mathrm{P}=0.6758)$.

2. The recurrence rate is significantly higher in group I ( 8 patients $\simeq 19.05 \%$ ) than group II $(1$ patient $\simeq 2.38 \%)(\mathrm{P}=0.0294)$. The patients with recurrent hernias were re-operated upon reinforcing the repair with double face proceed mesh, with no rerecurrence after repair during the follow-up period.

3. Lower skin flap necrosis occurred in 1 patient of groups II $(2.38 \%)$ who required debridement and skin graft when the infection resolved $(\mathrm{P}=1)$.

4. Minor skin necrosis of the wound occurred in 3 patients of group I (7.14\%) and in 2 patients of group II $(4.76 \%)$ which required repeated dressing and healed without further re-operation $(\mathrm{P}=1.0000)$.

5. The incidence of wound infection was higher in group II ( 5 patients $\simeq 11.9 \%$ ) than group I ( 2 patients $\simeq 4.76 \%$ ). However the difference was insignificant $(\mathrm{P}=0.4326)$.
These patients required daily dressings and broad spectrum antibiotic administration and healed without the need for re-operation.

6. The incidence of wound seroma was higher in group II ( 9 patients $\simeq 21.43 \%$ ) than group I (6 patients $\simeq 14.29 \%)$. However the difference was statistically insignificant $(\mathrm{P}=0.5700)$. These patient required repeated aspiration of the seroma except in 2 patients; one in each group who required re-insertion of suction drain for 10 days.

7. Stitch sinus occurred in 1 patient of group I (2.38\%) and 2 patients of group II $(4.76 \%)(\mathrm{P}=1.0000)$. The three patients required stitch sinus excision with good healing afterwards.

8. Deep venous thrombosis of lower limbs occurred in 1 patients of group I $(2.38 \%)$ and 1 patients of group II $(2.38 \%)$ $(\mathrm{P}=1.0000)$. No incidence of pulmonary embolism. Patients with DVT were successfully treated with S.C. Low molecular weight heparin then oral anticoagulants till complete re-canalization of the deep veins Table (5).

No mesh extrusion occurred in any patient of group II $(0 \%)$.

\section{Discussion:}

Open component separation for tensionfree approximation of fascial borders is increasingly used for repair of large mid line ventral hernias. ${ }^{12}$

In our study, we achieved closure of large fascial defects of the abdominal wall associated with large incisional hernias, by adopting the technique of component separation either alone in group I or combined with polypropylene mesh in group II.

The mean operative time was significantly shorter in group I $(165 \pm 24.09$ min. $)$ than group II (195.60 $\pm 19.42 \mathrm{~min}$. $)(\mathrm{P}<0.0001)$.

In a recent study the mean operative time for component separation was $143.27 \mathrm{~min}^{13}$

In our study, there was no intraoperative complications in both groups, except small intestinal injury in 1 patient of group I $(2.38 \%)$ which was repaired in 2 layers without any subsequent leakage $(\mathrm{P}=1)$.

In a study, an unavoidable enterotomies 


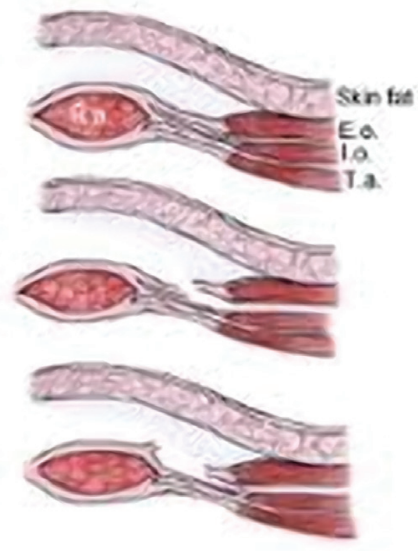

Figure (1): Diagram shows component separation technique.
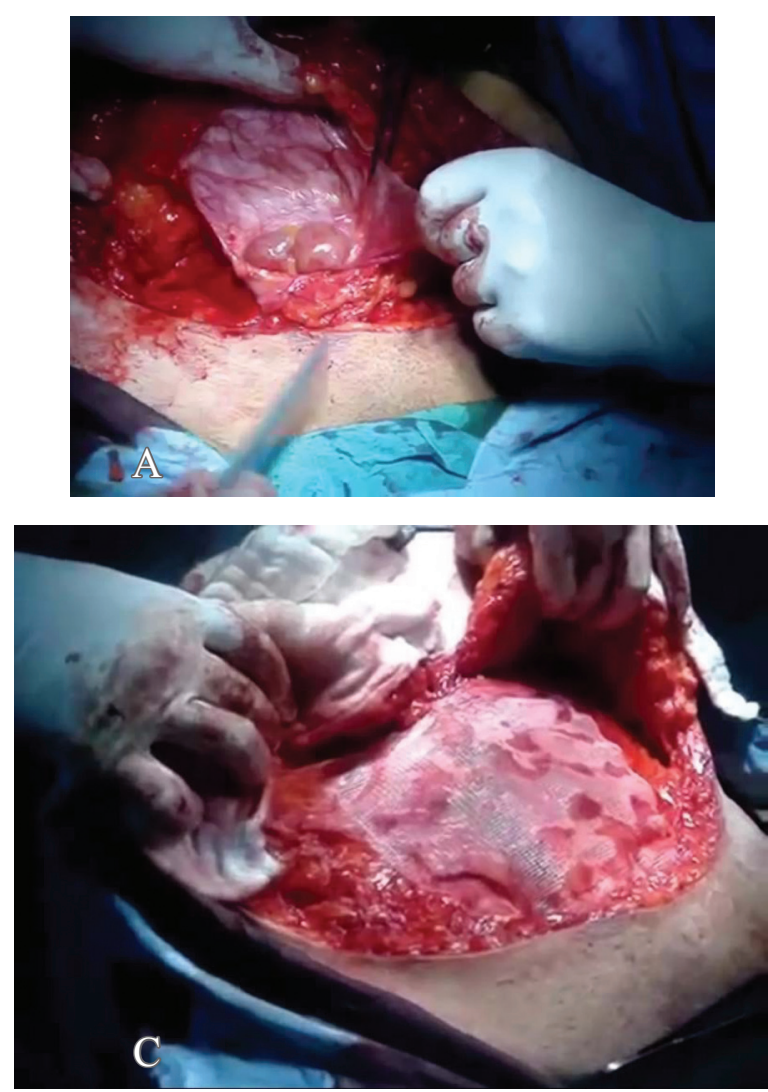
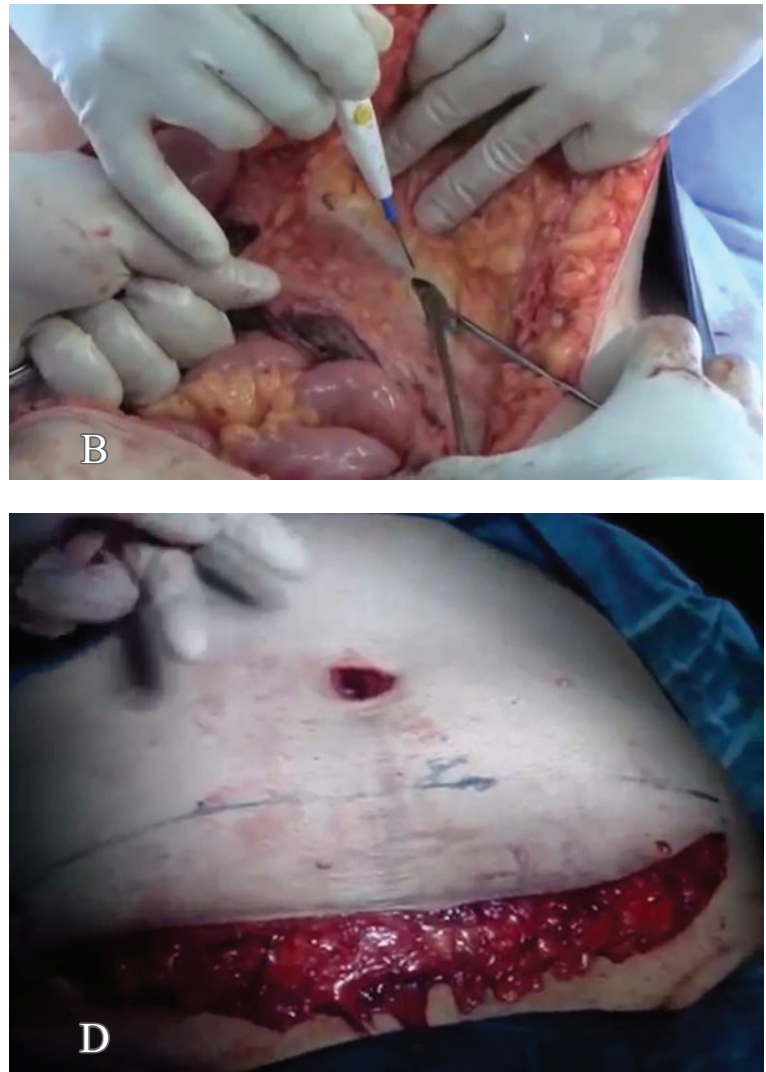

Figure (2): (a) shows big hernial defect, (b) incising the external oblique fascia and dissecting through the external oblique muscle until the internal oblique fascia was encountered. (c) plication of the midline then application of an onlay polypropylene mesh (d) excess skin and subcutaneous tissue was excised.

occurred in $3.6 \%$ of patients (all of which were repaired easily and without further complication) and unavoidable cystostomy in $1.2 \%{ }^{4}$

In our study, there was no manifestations of abdominal compartmental syndrome in any patient of both groups, and there was no statistically significant difference in the mean of the maximum recorded intra-abdominal pressure between both groups of patients $(\mathrm{P}=0.4733)$.

The Ramirez component separation operation can successfully repair hernias as large as $35 \mathrm{~cm}$ in transverse diameter achieving abdominal wall closure and no subsequent abdominal compartment syndrome. ${ }^{8}$ 
Table (1): Sex and mean age.

\begin{tabular}{|l|l|l|l|l|}
\hline \multicolumn{2}{|r|}{ Proup } & \multicolumn{1}{|c|}{ Group I } & \multicolumn{1}{c|}{ Group II } & \multirow{2}{*}{ P-value } \\
\hline \multirow{2}{*}{ Sex } & Female & $25(59.92 \%)$ & $27(64.29 \%)$ & \multirow{2}{*}{$0.6615337 \%$} \\
\cline { 2 - 4 } & Male & $17(40.48 \%)$ & $15(35.71 \%)$ & \\
\hline \multicolumn{2}{|l|}{ Mean age (years $\pm \mathrm{SD})$} & $45.45 \pm 8.39$ & $48-97 \pm 8.23$ & 0.05544 \\
\hline
\end{tabular}

Table (2): Mean operative time and mean blood loss.

\begin{tabular}{|c|c|c|c|}
\hline $\begin{array}{ll}\text { Parameter } & \text { Group } \\
\end{array}$ & Group I & Group II & P-value \\
\hline Mean operative time $(\min . \pm \mathrm{SD})$ & $165.00 \pm 24.09$ & $195.60 \pm 19.42$ & $<0.0001$ \\
\hline Mean blood loss $(\mathrm{mL} \pm \mathrm{SD})$ & $725.00 \pm 147.85$ & $773.81 \pm 141.09$ & 0.1255 \\
\hline
\end{tabular}

Table (3): Mean intra-abdominal pressure and mean VAS.

\begin{tabular}{|c|c|c|c|}
\hline Parameter $\quad$ Group & Group I & Group II & P-value \\
\hline Mean intra-abdominal pressure $(\mathrm{mmHg})$ & $7.2 \pm 1.72$ & $7.29 \pm 1.61$ & 0.4733 \\
\hline Mean VAS (1st 48 hours) & $6.36 \pm 1.27$ & $6.24 \pm 1.27$ & 0.6675 \\
\hline Mean VAS (3rd -7 th day) & $3.64 \pm 1.12$ & $3.24 \pm 0.93$ & 0.0758 \\
\hline
\end{tabular}

Table (4): Mean hospital stay and mean time to return to work.

\begin{tabular}{|c|c|c|c|}
\hline Parameter $\quad$ Group & Group I & Group II & P-value \\
\hline Mean hospital stay (days \pm SD) & $6.50 \pm 1.33$ & $6.50 \pm 1.84$ & 1 \\
\hline Mean time to return to work (days $\pm \mathrm{SD}$ ) & $23.17 \pm 4.46$ & $23.12 \pm 4.17$ & 0.9596 \\
\hline
\end{tabular}

Table (5): Post-operative complications.

\begin{tabular}{|l|l|l|l|}
\hline Complications & \multicolumn{1}{|c|}{ Group } & \multicolumn{1}{c|}{ Group II } & \multicolumn{1}{c|}{ P-value } \\
\hline Anaemia & $4(9.52 \%)$ & $2(4.76 \%)$ & 0.6758 \\
\hline Recurrence & $8(19.05 \%)$ & $1(2.38 \%)$ & 0.0294 \\
\hline Lower skin flap necrosis & $0(0 \%)$ & $1(2.38 \%)$ & 1.0000 \\
\hline Minor skin necrosis & $3(7.14 \%)$ & $2(4.76 \%)$ & 1.0000 \\
\hline Wound infection & $2(4.76 \%)$ & $5(11.90 \%)$ & 0.4326 \\
\hline Wound seroma & $6(14.29 \%)$ & $9(21.43 \%)$ & 0.5700 \\
\hline Stitch sinus & $1(2.38 \%)$ & $3(4.76 \%)$ & 1.0000 \\
\hline DVT & $1(2.38 \%)$ & $1(2.38 \%)$ & 1.0000 \\
\hline
\end{tabular}

In our study, there was no significant difference in the post-operative pain severity between both groups during the $1^{\text {st }} 48$ hours $(\mathrm{P}=0.6675)$, while from the $3^{\text {rd }}$ to the $7^{\text {th }}$ post-operative day the pain severity was less in group II than group I without reaching statistical significance $(\mathrm{P}=0.0758)$.

In our study, there was a significantly higher recurrence rate in the component separation without mesh group (group I: 8 
patients $\simeq 19.05 \%$ ) than CS with mesh (group II: 1 patient $\simeq 2.38 \%)(\mathrm{P}=0.0294)$.

In a meta-analysis of 21 studies including 927 patients, comparing open component separation, minimally invasive component separation (MICS), mesh repair, and suture repair for complex ventral hernia repair, the recurrence rate was $21 \%, 17 \%, 33 \%, 24 \%$ for open CS, MICS, mesh repair and suture repair respectively. Open CS with mesh seemed to have lower recurrence than open CS alone (16.7 vs $27 \%$ respectively). ${ }^{14}$

In another meta-analysis, the incidence of recurrent hernia was 13\% after endoscopic component separation and $16 \%$ after open component separation (odds ratio $0.76,95 \%$ confidence interval $0.29-1.98, \mathrm{P}=0.57) .{ }^{12}$

In another study 8 of 82 operations had a subsequent hernia recurrence $(9.8 \%){ }^{4}$

Another study reported $8.3 \%$ hernia recurrence rate after component separation for the repair of large abdominal wall hernia following bariatric surgery. ${ }^{11}$

However, in a relatively large series of 43 patients, de Vries Reilingh and colleagues ${ }^{15}$ were unable to reproduce the good results of Ramirez and recorded recurrent hernia in $32 \%$ of patients at 15 months follow-up.

In our study, other complications included anemia corrected by blood transfusion in 4 patients of group I (9.52\%) and 2 patients of group II $(9.76 \%)(\mathrm{P}=0.6758)$; lower skin flap necrosis in 1 patient of group I (2.38\%) which required debridement and skin graft $(\mathrm{P}=1.000)$; minor skin wound necrosis in 3 patients of group I (7.14\%) and in 2 patients of group II $(4.76 \%)(\mathrm{P}=1.000)$; wound infection in 2 patients of group I $(4.76 \%)$ and 5 patients of group II $(11.90 \%)(\mathrm{P}=0.4326)$; wound seroma in 6 patients of group I (14.29\%) and 9 patients of group II $(21.43 \%)(\mathrm{P}=0.5700)$; stitch sinus in 1 patient of group I $(2.38 \%)$ and 2 patients of group II $(4.76 \%)(\mathrm{P}=1.000)$; and deep venous thrombosis of calf veins in 1 patient of group I (2.38\%) and 1 patient of group II (2.38\%).

In a meta-analysis, 6 cases of enterocutaneous fistula occurred in open component separation, 1 wound dehiscence in open component separation, 17 superficial infection and 21 mesh infection in open CS, 8 mesh infections with mesh repair and 8 wound infections in laparoscopic CS. ${ }^{14}$

In another recent meta-analysis, the incidence of wound complication comprising surgical site infection, subcutaneous abscess, seroma, skin dehiscence, cellulitis, and fistula was significantly less after endoscopic CS than open CS (odds ratio 0.27, 95\% confidence interval $0.12-0.58, \mathrm{P}<0.001) .12$

In another study, of open CS for the repair of large abdominal wall hernias following bariatric surgery, there was a high rate of minor or major wound complications $(50 \%)$; however all of these wounds closed without additional procedures. ${ }^{11}$

In another study of 82 operations, major complications occurred in 3 patients and included 1 enterocutaneous fistula (1.2\%), 1 patient with notable skin flap necrosis requiring operative debridement and subsequent split-thickness skin grafting $(1.2 \%)$, and 1 patient who experienced an extrusion of polypropylene mesh 2 years after repair $(1.2 \%)$. The most common minor complication was minor skin necrosis or dehiscence requiring local wound care only, occurring in 12 patients $(14.6 \%)$. Cellulitis requiring either intravenous or oral antibiotics occurred in 8 patients $(9.7 \%)$. Infected fluid collections requiring local wound care and antibiotics occurred in 7 patients $(8.5 \%)$ uninfected seroma or hematoma occurred in 4 patients $(4.8 \%){ }^{4}$

In our study, there was no statistically significant difference in the mean hospital stay $(\mathrm{P}=1.000)$ and mean time to return to work between both groups of patients ( $\mathrm{P}=0.9596 \%)$.

\section{Conclusion:}

Large ventral abdominal wall incisional hernia need a multidisciplinary team of general and plastic surgeons to offer an efficient adhesolysis preventing further adhesive intestinal obstruction, and to offer a tension-free abdominal wall repair without increasing the intraabdominal pressure to avoid abdominal compartmental syndrome.

The abdominal component separation 
offers a good technique for tension-free abdominal wall repair for incisional hernia with large abdominal defects.

Further inforcement of the component separation technique of fascial repair with prosthetic mesh reduces the chance for hernial recurrence without significantly increasing the incidence of intra- or post-operative complications or the length of hospital stay.

\section{Reference:}

1- Rudmik LR, Schieman C, Dixon E, Debru E: Laparoscopic incisional hernia repair: A review of the literature. Hernia 2006; 1: 110-119.

2- Wong SY, Kingsnorth AN: Prevention and surgical management of incisional hernias. Int J Surg Invest 2001, 3: 407-414.

3- Van't Riet M, deVos van Steenwijk PJ, Bonjer HJ, Steyerberg EW, Jeekel J: Incisional hernia after repair of wound dehiscence: Incidence and risk factors. Ann Surg 2004; 70: 281-286.

4- Robertson JD1, de la Torre JI, Gardner PM, Grant JH, Fix RJ, Vásconez LO: Abdominoplasty repair for abdominal wall hernias. Ann Plast Surg 2003; 51:10-16.

5- Girotto JA, Ko MJ, Redett R, Muehlberger T, Talamini M, Chang B: Closure of chronic abdominal wall defects: A long-term evaluation of the components separation method. Ann Plast Surg 1999; 42: 385-394.

6- Korenkov M, Paul A, Sauerland S, Neugebauer E, Arndt M, Chevrel JP, et al: Classification and surgical treatment of incisional hernia. Results of an experts' meeting. Lang Arch Surg 2001; 386: 65-73.

7- Ramirez OM, Ruas E, Dellon AL: Components separation method for closure of abdominal-wall defects: An anatomic and clinical study. Plast Reconstr Surg 1990; 86:
519-526.

8- Kingsnorth A: The management of incisional hernia. Ann $R$ Coll Surg Engl 2006; 88: 252-260.

9- Munegato G, Brandolese R: Respiratory physiopathology. In: Surgical repair for large incisional hernias of the abdominal wall. 2001; 192: 298-304.

10- Shestak KC, Edington HJD, Johnson RR: The separation of anatomic components technique for the reconstruction of massive midline abdominal wall defects: Anatomy, surgical technique, applications and limitations revisited. Plat Reconst Surg 2000; 105: 731-738.

11- Borud LJ, Grunwaldt L, Janz B, Mun E, Slavin SA: Components separation with abdominal wall plication for repair of large abdominal wall hernias following bariatric surgery. Plat Reconst Surg 2007; 119: 1792-1728.

12- Jensen KK, Henriksen NA, Jorgensen LN: Endoscopic component separation for ventral hernia causes fewer wound complications compared to open components separation: A systematic review and meta-analysis. Surgical Endoscopy 2014; 28: 3046-3052.

13- Shih PK: Abdominal wall closure: Component separation versus partition technique. Hernia 2014; DOI.10.1007/ S10029-014-1238-4.

14- Winnil TMY, William H, David OW, Charles HS: Comparison of outcome after mesh-onlay repair, laparoscopic component separation, and open component separation. Annals of Plastic Surgery 2011; 66(5): 551-556.

15- De Viries RTS, van Goor H, Rosman C, Benselmans MHA, de Jong D, van Niecewenhoven EJ, et al: Components separation technique for the repair of large abdominal wall hernias. $J$ Am Coll Surg 2003; 196: 32-37. 